\section{Histone deacetylase-associating Atrophin proteins are nuclear receptor corepressors}

\author{
Lei Wang, ${ }_{1}^{1}$ Harini Rajan, ${ }^{1}$ Jeffrey L. Pitman, ${ }^{2}$ \\ Michael McKeown, ${ }^{2}$ and Chih-Cheng Tsai ${ }^{1,3}$ \\ ${ }^{1}$ Department of Physiology and Biophysics, University of \\ Medicine and Dentistry of New Jersey-Robert Wood Johnson \\ Medical School, Piscataway, New Jersey 08854, USA; \\ ${ }^{2}$ Department of Molecular Biology, Cell Biology, and \\ Biochemistry, Brown University, \\ Providence, Rhode Island 02912, USA
}

\begin{abstract}
Drosophila Tailless (Tll) is an orphan nuclear receptor involved in embryonic segmentation and neurogenesis. Although Tll exerts potent transcriptional repressive effects, the underlying molecular mechanisms have not been determined. Using the established regulation of knirps by $t 11$ as a paradigm, we report that repression of knirps by Tll involves Atrophin, which is related to vertebrate Atrophin-1 and Atrophin-2. Atrophin interacts with Tll physically and genetically, and both proteins localize to the same knirps promoter region. Because Atrophin proteins interact with additional nuclear receptors and Atrophin-2 selectively binds histone deacetylase 1/2 (HDAC1/2) through its ELM2 (EGL-27 and MTA1 homology 2)/SANT (SWI3/ADA2/N-CoR/TFIIIB) domains, our study establishes that Atrophin proteins represent a novel class of nuclear receptor corepressors.
\end{abstract}

Supplemental material is available at http://www.genesdev.org.

Received November 18, 2005; revised version accepted December 30, 2005.

Nuclear receptors (NRs) comprise one of the largest known families of eukaryotic transcription factors (Mangelsdorf and Evans 1995). The majority of identified NRs are "orphans," without known ligands. Many of these orphan NRs are conserved between vertebrates and flies (King-Jones and Thummel 2005), which makes the fly an ideal model system to study their properties. A major function of NRs is transcriptional repression. For unliganded members of subfamily 1 of the NR superfamily (NR1), such as thyroid hormone receptor (TR), retinoic acid receptor (RAR), and ecdysone receptor (EcR), their repression is often mediated by corepressors from the SMRT/N-CoR/SMRTER family (Chen and Evans 1995; Horlein et al. 1995; Tsai et al. 1999; Privalsky 2004; Tsai and Fondell 2004), which share a conserved SANT (SWI3/ADA2/N-CoR/TFIII-B) domain (Aasland et al. 1996) and a repeated arginine-glutamic acid/(RERE) stretch (Tsai et al. 1999). The conservation of the SANT

[Keywords: Atrophin; Tailless; nuclear receptor; ELM2 domain; SANT domain; histone deacetylase]

${ }^{3}$ Corresponding author.

E-MAIL tsaich@umdnj.edu; FAX (732) 235-5038.

Article published online ahead of print. Article and publication date are at http://www.genesdev.org/cgi/doi/10.1101/gad.1393506. domain, which is present in several other chromatin and transcriptional regulators (Aasland et al. 1996), and of the RERE stretch in SMRT/N-CoR/SMRTER suggests that these two domains are functionally significant. The SANT domain appears to enable various transcriptional regulators to carry out different chromatin-modifying tasks in a context-dependent manner. For example, (1) a SANT domain of ADA2 and SMRT interacts with histone tails (Boyer et al. 2002; Yu et al. 2003); (2) a SANT domain of SMRT binds to and activates histone deacetylase 3 (HDAC3) (Guenther et al. 2001; Yu et al. 2003; Codina et al. 2005); and (3) a SANT domain enables CoREST, a corepressor of REST, to stimulate histone demethylation activity for LSD1 (Shi et al. 2005).

Tailless (Tll) is an orphan NR2 protein first identified as a terminal gap gene product involved in Drosophila segmentation (Pignoni et al. 1990). Tll is expressed at both anterior and posterior poles of the embryo. It strongly represses the transcription of another gap gene, knirps (kni) (Pankratz et al. 1989). Similar repression of kni can be achieved by Tlx, the vertebrate cognate of Tll, when it is ectopically expressed in Drosophila embryos (Yu et al. 1994). The similar transcriptional properties of Tll and Tlx imply that both proteins recruit similar corepressor(s) to repress kni in Drosophila. Thus far, no strong candidates for corepressors of Tll/Tlx have been identified.

In this study, we report the identification of Atrophin (Atro; also known as Grunge), which encodes a SANT domain, a RERE stretch, and an ELM2 (EGL-27 and MTA1 homology 2) domain, as an interacting factor of Tll. Mutations of Atro have been shown to cause a variety of patterning defects in Drosophila (Erkner et al. 2002; Zhang et al. 2002; Fanto et al. 2003; Kankel et al. 2004). Because Atro is related to two vertebrate proteins, Atrophin-1 (Atr1), a dentatorubral pallidoluysian atrophy (DRPLA) protein (Koide et al. 1994; Nagafuchi et al. 1994), and Atrophin-2 (Atr2; also called RERE) (Yanagisawa et al. 2000; Waerner et al. 2001), we further investigated the relationships between Atro proteins and nuclear receptors in both Drosophila and vertebrate cells. Our study reveals that Atro proteins represent a new class of nuclear receptor corepressors, whose ELM2 and SANT domains play a central role in mediating their HDAC activity.

\section{Results and Discussion}

\section{Identifying Atro as a Tll-interacting factor}

Since SMRT is a transcriptional corepressor for many NRs (Privalsky 2004) and SMRTER is the Drosophila cognate of SMRT (Tsai et al. 1999), our first step in identifying Tll/Tlx-interacting corepressors was to test whether Tll and Tlx interact with SMRT and SMRTER. Using yeast two-hybrid assays, we found that, whereas EcR, TR, and RAR interact with both SMRTER and SMRT, both Tll and Tlx fail to interact with SMRTER or SMRT (Supplementary Fig. 1).

To find potential corepressors of Tll/Tlx, we turned to a yeast two-hybrid screen, in which a Tll-expressing bait construct was deployed against a Drosophila embryonic library. A positive clone was identified, whose insert codes for the (1301-1966) region of Atro. This clone was 
selected for further investigation for several reasons: (1) In yeast, this clone also interacts strongly with chick and human Tlx, but not with RAR or TR (Fig. 1A); (2) Atro encodes a SANT domain, a RERE stretch, and an ELM2 domain; (3) Atro is a transcriptional corepressor of the Drosophila segmentation gene even-skipped (Zhang et al. 2002); (4) two Atro-related proteins, Atr1 and Atr2, exist in vertebrates (Koide et al. 1994; Nagafuchi et al. 1994; Yanagisawa et al. 2000; Waerner et al. 2001); and (5) Atr2 interacts with HDAC1 in mouse embryos (Zoltewicz et al. 2004). These properties of Atro proteins highlight the possibility that they are corepressors for Tll and Tlx.

\section{$L B D$ is required for Tll/Tlx to interact with Atro}

To determine which region in Tll is required for Atro association, we generated the series of truncated Tll expression constructs shown in Figure $1 \mathrm{~B}$ and tested their interactions with Atro in yeast. The (192-452) region of Tll was found to be sufficient to mediate its interaction with Atro. Since this region of Tll harbors its ligandbinding domain (LBD), it suggested to us that an intact LBD is required for Tll to bind Atro. Indeed, no associa-

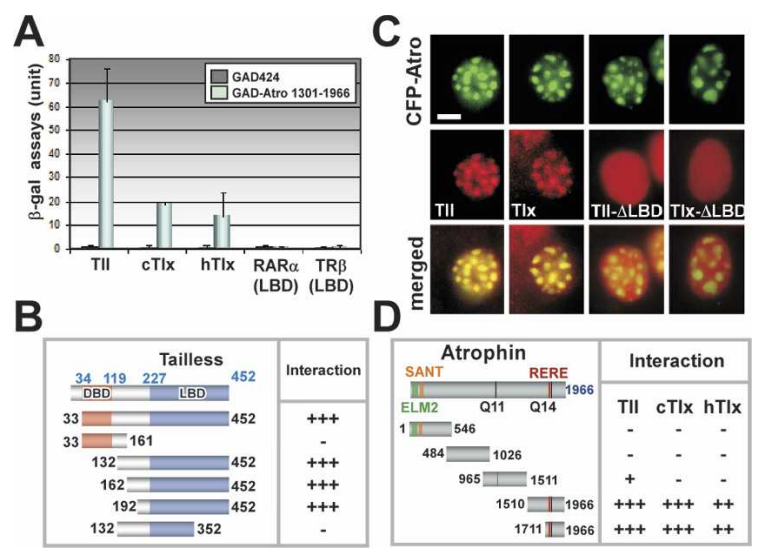

Figure 1. Atro interacts with Tll through its LBD. $(A)$ Liquid $\beta$-galactosidase assay, showing the interaction between Tll or Tlx and an isolated Atro clone from the yeast two-hybrid screen. Yeast cells were transformed with different combinations of plasmids expressing each of the indicated NRs (in Gal4-DNA-binding domain fusion [GBT]) and the isolated Atro clone (in Gal4-activating domain fusion [GAD]). The tested NRs include Tll, chick Tlx (cTlx); human Tlx (hTlx), and the LBD of human TR $\beta$ and RAR $\alpha$. Protein-protein interaction was measured in $\beta$-gal units (mean $+\mathrm{SD}, n=3)$. $(B)$ Results from yeast two-hybrid assays showing a LBD-dependent interaction between Tll and Atro. Y190 cells were transformed with serial GBTbased plasmids expressing the indicated regions of Tll, and with the isolated Atro clone. Strong positive and negative interactions are marked with +++ and -, respectively. (DBD) DNA-binding domain. (C) Immunostaining showing a LBD-dependent colocalization of Tll or Tlx and Atro in cultured human cells. HEK293 cells were transfected with different combinations of plasmids expressing cyan fluorescent protein $(\mathrm{CFP})$-tagged Atro, and Flag-tagged Tll, Tlx, Tll $\Delta \mathrm{LBD}$, or Tlx $\Delta \mathrm{LBD}$. Flag fusion proteins were detected with antiFlag antibody (Texas-Red). (Tll $\Delta$ LBD) Tll(33-191); (Tlx $\Delta$ LBD) Tlx $(1-$ 163). Bar, $10 \mu \mathrm{M}$. (D) Results from yeast two-hybrid assays showing the regions in Atro responsible for Tll or Tlx interactions. Yeast cells were transformed with GBT-based Tll- or Tlx-expressing plasmids, along with GAD-based plasmids expressing the indicated Atro fragments. The interactions, based on the intensity of the reporter activity, are shown as,,++++++ , and - , respectively. The ELM2, SANT, RERE, and glutamine-repeat (Q) regions in Atro are indicated. tion between Tll variants lacking an intact LBD [e.g., Tll(33-161) or Tll(132-352)] and Atro could be detected.

A LBD-dependent interaction between Tll and Atro was further confirmed in human cells by using an immunostaining approach. CFP-tagged Atro (CFP-Atro) localizes to subnuclear regions when expressed in cells (Fig. 1C). This nuclear focal pattern of Atro resembles the nuclear pattern known for Atr2 (Yanagisawa et al. 2000; Waerner et al. 2001). Expressing Atro with Tll or Tlx in the same cells alters the nuclear distribution of Tll and Tlx: Both Tll and Tlx shift from their evenly distributed nuclear patterns (Supplementary Fig. 2) to punctate nuclear patterns virtually identical to that displayed by CFP-Atro. Deleting the LBD from Tll and Tlx abrogates their localization to Atro-positive nuclear foci, confirming that Atro-Tll/Tlx interactions are mediated through the LBD of Tll and Tlx.

We next mapped the regions in Atro responsible for Tll or Tlx interaction, using the serial deletion Atro constructs shown in Figure 1D. Two regions in Atro were found to mediate its interaction with Tll: Atro(9651511) interacts weakly with Tll, whereas Atro(17111966) interacts strongly with both Tll and Tlx. The latter finding is of great interest, since the 1711-1966 region of Atro contains sequences conserved in the C-terminal regions of vertebrate Atr1 and Atr2. This correlation prompted us to investigate whether Atr1 and Atr2 interact with Tll or Tlx.

\section{Interactions between Atro and Tll/Tlx depend} on a conserved Atro-box

Accordingly, two constructs expressing the C-terminal regions of Atr1 and Atr2 were generated and tested individually against Tll- or Tlx-expressing plasmids. As expected, both Atr1(846-1191) and Atr2(1224-1566), like Atro(1711-1966), interact strongly with Tll and Tlx in yeast (Fig. 2A), confirming that all Atro proteins are commonly targeted by Tll/Tlx.

Because the mapped Tll/Tlx-interacting regions in Atro, Atr1, and Atr2 share a stretch of highly conserved residues (Fig. 2B), we next investigated whether mutations created within this region, which we call the Atrobox, affect Tll/Tlx interaction. Indeed, substitution of two leucine residues with alanine abolishes the interaction between Atro(1711-1966) and Tll or Tlx in yeast (Fig. 2C). Atro-Tll/Tlx interactions are, therefore, in part mediated through the Atro-box.

\section{Atro proteins interact with additional nuclear receptors}

T1l/Tlx belong to the NR2 subfamily of the NR superfamily. The similarity shared by members of the NR2 subfamily suggests that additional NR2 proteins may interact with Atro proteins as well. We tested this possibility first with GST pull-down assays, in which several ${ }^{35}$ S-methionine-labeled NR2 and NR1 proteins were tested for their interactions with GST or GST-Atro fusion proteins. As shown in Figure 2D, Atro proteins specifically bind Tll, Tlx, human chicken ovalbumin upstream promoter-transcription factor (COUP-TF), and Seven-Up1 (SVP1) (the Drosophila COUP-TF homolog), but not TR $\beta$ and Ultraspiracle (USP). A similar interacting profile was observed between Atro proteins and COUP-TF or SVP1 in yeast (Supplementary Fig. 3). 


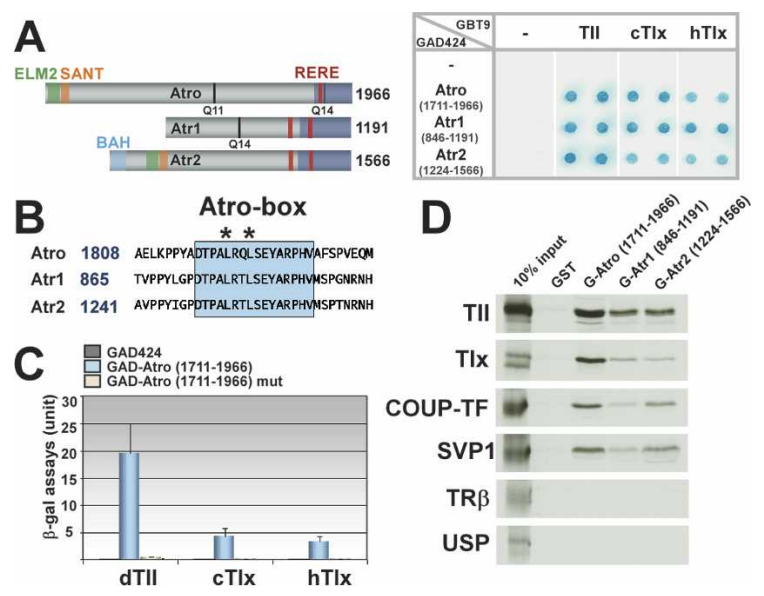

Figure 2. Atro proteins interact with NR2 proteins. $(A)$ Yeast twohybrid results showing the cross-species interactions between Drosophila and vertebrate Atro and Tll proteins. Y190 cells were transformed with plasmids expressing the indicated regions of Atro, Atrl, or Atr2, and plasmids expressing Tll, cTlx, or hTlx. The mapped Tll/Tlx-interacting regions are dark blue. Two wells were used for each experiment. Positive interaction is revealed by the blue (X-gal) reaction. (B) Alignment of sequences showing a highly conserved motif, termed the Atro-box, found in Atro, Atr1, and Atr2. The amino acid numbers for each Atro protein are indicated. Asterisks indicate the sites where mutations (leucine to alanine) were introduced. $(C)$ Liquid $\beta$-galactosidase assays showing the lack of interaction between Tll or Tlx and Atro(1711-1966) with a mutated Atrobox. Protein-protein interaction was measured in $\beta$-galactosidase units (mean $+\mathrm{SD}, n=3)$. (D) GST pull-down assays showing the preferential binding of Atro proteins to NR2 proteins. Bacterially expressed GST or GST fusion proteins were mixed with in vitro translated ${ }^{35} \mathrm{~S}$-methionine-labeled NRs, including Tll, hTlx, COUPTF, SVP1, TR $\beta$, and USP. The bound proteins were resolved by gel electrophoresis.

Therefore, Atro proteins do not interact with all NRs; rather, they preferentially bind a subset of NR2, including Tll, Tlx, SVP1, and COUP-TF.

\section{Atro and tll interact genetically to repress kni expression in Drosophila}

Having demonstrated that Atro physically interacts with various NRs, we turned to the biological relevance of these interactions. In this study, we focus on the in vivo relationship between Atro and Tll in flies by exploiting the known role of Tll in the segmentation process during Drosophila early embryogenesis. At this stage, Atro is expressed as a nuclear protein throughout the embryos (Erkner et al. 2002; Zhang et al. 2002). Consistent with previous observations that $t 11$ represses kni expression at the posterior end of the embryo (Pankratz et al. 1989), in situ hybridization for $t 11^{1}$ embryos shows a posterior expansion of kni stripe, especially in the ventral region of the embryos (Fig. 3A). Removal of zygotic Atro alone, as in the P-element excision line Atro ${ }^{35}$ (Erkner et al. 2002), does not cause such expansion, due to the presence of maternally deposited Atro. When maternal alone or both maternal and zygotic Atro are depleted using the dominant female sterile-FLP method (Chou and Perrimon 1992), however, kni expression expands posteriorly in embryos. Because mutations of Atro and tll alter kni expression similarly, these in vivo observations suggest that Atro and tll are involved in overlapping transcriptional pathways.
To address the genetic interaction between $t 11$ and Atro further, we took advantage of the hypomorphic nature of the $t 11^{1}$ allele (Strecker et al. 1986) and asked whether the $t 11^{1}$-mediated phenotype is aggravated by additional Atro mutation. Specifically, we investigated whether the observed posterior expansion of kni stripe in t11 ${ }^{1}$ embryos becomes more prominent when zygotic Atro is removed. Accordingly, we generated a $t^{1} 1^{1}$, Atro $^{35}$ double-mutant fly line, in which both $t 11^{1}$ and $A t r O^{35}$ alleles were recombined to the same chromosome, and tested kni expression in the resulting homozygous mutant embryos. Indeed, a further posterior expansion of kni stripe was observed in $t 11^{1}$, Atro $^{35}$ double-mutant embryos, mimicking that found in $t 11^{e}$ embryos (note that $t 1 l^{e}$ is a deficiency line, in which the $t l 1$ gene unit is

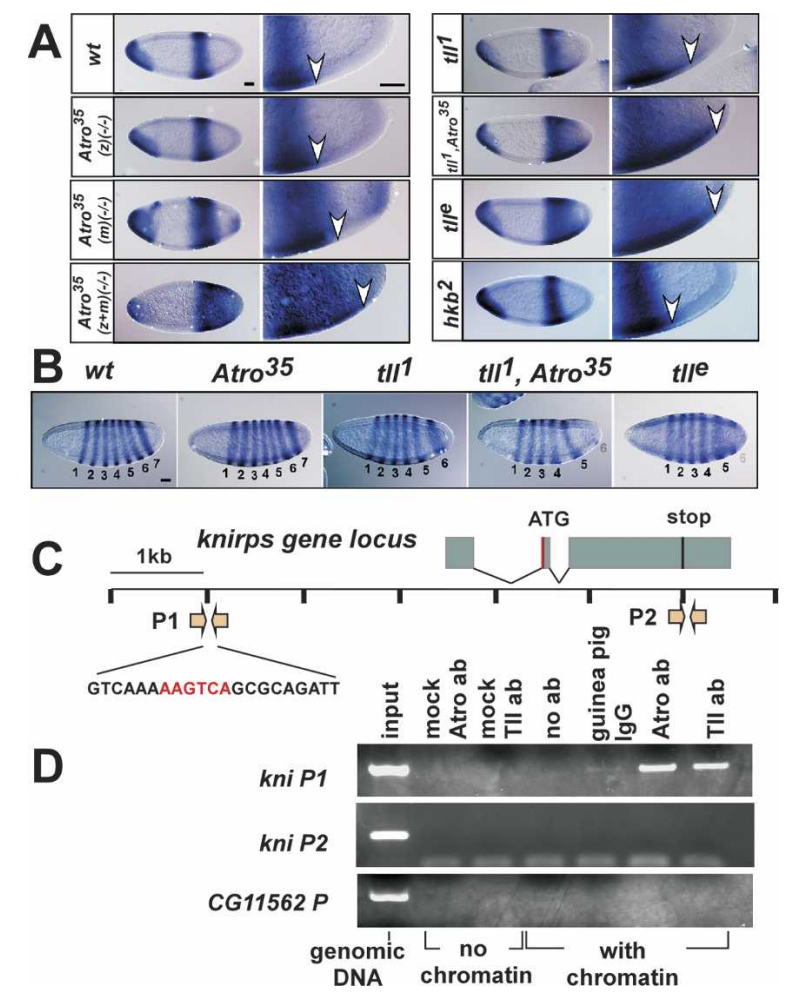

Figure 3. Atro and tll act together to repress kni. (A) In situ hybridization experiments showing the expression patterns of kni in each of the indicated mutant embryos. $(z)^{-1-}$ or $(\mathrm{m})^{-1-}$ indicates that only the zygotic or the maternal contribution of Atro is depleted, $(\mathrm{m}+\mathrm{z})^{-/-}$indicates that both maternal and zygotic Atro are depleted. $t 11^{1}$ is a hypomorphic allele; $t 1 l^{e}$ is a deficiency line; $\operatorname{Atro}^{35}$ is a P-element excision line; $t 11^{1}$, Atro $^{35}$ is a double-mutant fly line. The posterior margin of each kni stripe on the ventral side of the embryo is marked with an arrowhead. The enlarged image on the right corresponds to the posterior-ventral region of the embryo on the left. All embryos are shown with their anterior to the left and dorsal side up. Bar, $30 \mu \mathrm{M}$. $(B)$ In situ hybridization experiments showing the expression patterns of $f t z$ in each of the indicated embryos. The pair-rule stripes of $f t z$ from anterior to posterior are labeled from 1 to 7. (C) Schematic diagram showing the kni gene locus and two regions targeted for ChIP assays. The two tested regions are labeled P1 and P2. A Tll-binding site (in red) and its flanking sequences in P1 are shown. $(D)$ ChIP assays showing the specific binding of Atro and Tll to the P1 region in the kni promoter. Assays were performed on 0 - to 4-h-old wild-type embryos with the indicated antibodies or IgG. CG11562 is a randomly chosen gene whose promoter was used as a negative control. In "mock" experiments, no chromatin was added. 
entirely deleted). We therefore conclude that Atro is required for Tll to repress kni.

Since Atro is a binding factor of another terminal gap gene product, Huckebein (Hkb) (Zhang et al. 2002), we additionally examined the expression of $\mathrm{kni}$ in $h \mathrm{~kb} b^{2}$ mutant embryos. No significant posterior expansion of kni was observed, therefore indicating that the repression of kni in the posterior-terminal region primarily results from the combined effect of Tll and Atro.

The genetic interaction between tll and Atro was further assessed by monitoring the expression of the pairrule gene fushi tarazu $(f t z)$ in the posterior region of the mutant embryos discussed above. In wild-type and in Atro ${ }^{35}$ zygotic mutant embryos, $f t z$ is expressed as seven stripes in the central region (Fig. 3B). In $t 11^{1}$ embryos, however, the posterior stripes of $f t z$ (mostly the fifth, sixth, and seventh stripes) shift toward the posterior end. In the most severely affected $t 11^{1}$ embryos, the seventh stripe of $f t z$ is lost. This altered $f t z$ pattern is known to be the consequence of cell fate changes, partly owing to the posterior expansion of $\mathrm{kni}$, when $t \mathrm{ll}$ is mutated (Mahoney and Lengyel 1987). In $t 11^{1}$, Atro ${ }^{35}$ double-mutant embryos and in $t 11^{e}$ embryos, additional loss of the sixth stripe of $f t z$ was observed. Because the cell fate change is more pronounced in $t 11^{1}$, Atro ${ }^{35}$ double mutants than in $t 11^{1}$ or $A t r o{ }^{35}$ embryos, we conclude that Atro participates with Tll in determining posterior-terminal cell fates in early Drosophila embryos.

\section{Atro is recruited to the $\mathrm{kni}$ promoter}

To verify the involvement of Atro in the regulation of kni by Tll at the chromatin level, we carried out chromatin immunoprecipitation (ChIP) assays for 0- to 4-hold Drosophila embryos using Atro antibody, Tll antibody, and control IgG, respectively. The immunoprecipitated (IP) chromatin was subjected to PCR using primers corresponding to two separate regions, $\mathrm{P} 1$ and $\mathrm{P} 2$, in the kni gene (Fig. 3C), and a region in a randomly selected control (CG11562) promoter. In the kni promoter, P1 resides $2.5 \mathrm{~kb}$ upstream of the transcription initiation site and has a defined Tll-binding site (Pankratz et al. 1992). P2 corresponds to the 3' untranslated region of the kni gene, where no Tll-binding site is found.

Our in vivo ChIP assays revealed that both Atro and Tll antibodies, but not the control IgG, specifically precipitated chromatin that harbors the P1 site, but not chromatin containing P2 or the CG11562 promoter (Fig. $3 \mathrm{D})$. These results establish that Atro, by forming protein complexes with Tll, is present naturally on the kni promoter.

\section{Atro and Atr2, but not Atr1, exert potent HDAC activities}

Many transcriptional corepressors, including SMRT and $\mathrm{N}$-CoR, are associated with HDAC activity. Because our results indicate that Atro proteins are corepressors of Tll/Tlx, we further investigated (1) whether Atro proteins also show HDAC activity; (2) whether Atro proteins bind selected HDACs; and, if so, (3) which regions/ domains in Atro proteins mediate their HDAC binding. To address these interconnected questions, fluorometric HDAC assays and Western blot analysis were performed on protein complexes immunoprecipitated by Flagtagged Atro, Atr1, Atr2, or truncated Atr2 variants ex- pressed in HEK293 cells. In parallel experiments, Flag and Flag-SMRT were used as a negative and a positive control, respectively. The expression of tested Flag fusion proteins was first examined using Western blot analysis (Fig. 4A, right panel).

As expected, Flag-SMRT is associated with potent HDAC activity that is sensitive to trichostatin A (TSA), an HDAC inhibitor (Fig. 4B). Robust levels of TSA-sensitive HDAC activity were also observed for both Atro and Atr2, confirming that both proteins' properties involve HDACs. To our surprise, Atr1 displays no prominent HDAC activity. Since Atrl lacks the conserved ELM2 and SANT domains found in the N-terminal regions of Atr2 and Atro, we suspected that the missing N-terminal region in Atrl might be important for the HDAC activity of Atro proteins.

\section{ELM2/SANT domains mediate HDAC activity and HDAC1/2 association}

To determine whether the HDAC activity of Atro or Atr2 depends on its $\mathrm{N}$-terminal region, the BAH (Bromo adjacent homology), the ELM2, and the SANT domains in this region of Atr2 were deleted sequentially (Fig. 4A, lanes 5-7). Note that the BAH domain is absent in Atro. Whereas Atr2 $\triangle \mathrm{BAH}$ still exerts a robust level of HDAC activity, a dramatic reduction of HDAC activity was ob-

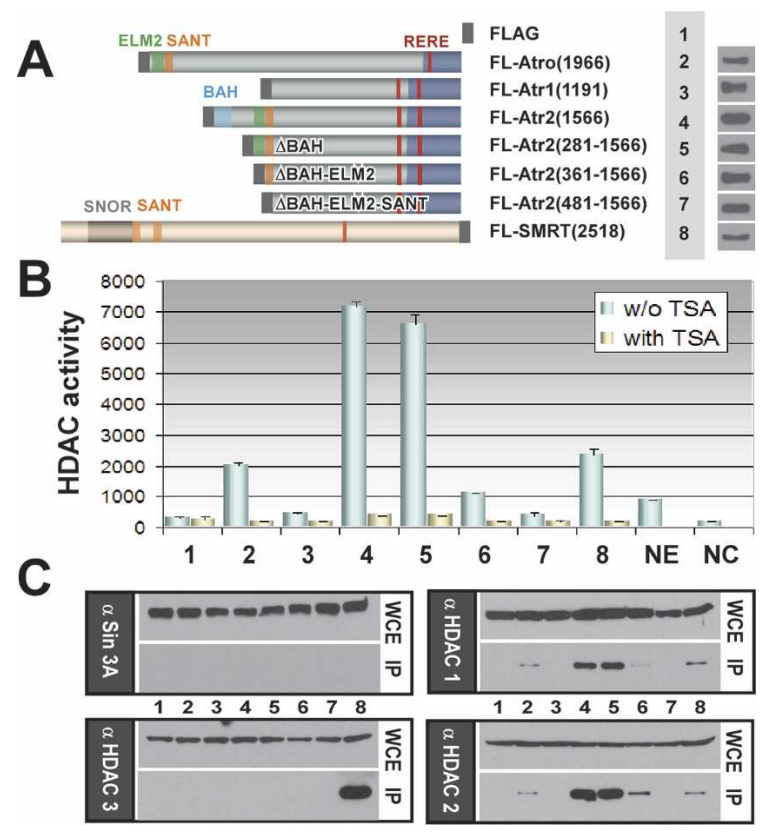

Figure 4. The ELM2/SANT domains mediate HDAC activity and HDACl/2 association. (A) Diagram showing the Flag-tagged proteins used in the HDAC assays and coimmunoprecipitation experiments. The functional domains in Atro proteins and the SNOR (SMRTER/SMRT/N-CoR) domain in SMRT are labeled with different colors. The Flag tag is shown as a gray box. The expression of proteins used for both assays is shown on the right. $(B)$ Chart showing the HDAC activity associated with each tested Flag fusion protein corresponding to those shown in A. TSA was used at the concentration of $1 \mu \mathrm{M}$. (NE) HeLa nuclear extracts; (NC) negative control (solutions without input protein). (C) Western blot analysis showing the preferential binding of Atro and Atr2 to HDACl and HDAC2. IP protein complexes associated with each Flag fusion protein were probed with the indicated antibodies. (WCE) Whole-cell extracts; (IP) immunoprecipitated proteins. 
served with Atr2 $\triangle \mathrm{BAH}-\mathrm{ELM} 2$ (Fig. 4B). A further deletion of the SANT domain, Atr2 $\triangle B A H-E L M 2-S A N T$, causes a complete loss of HDAC activity, indicating that both the ELM2 and SANT domains are central to Atr2's HDAC activity.

We next investigated which HDACs Atro proteins interact with, and whether Atr2's association with HDACs involves its ELM2/SANT domains. Protein complexes immunoprecipitated by Flag-tagged Atro proteins and Atr2 variants were examined by Western blot for a panel of potential associating proteins, including HDAC1, HDAC2, HDAC3, and Sin3A (Fig. 4C). Sin3A was not detected in any of the IP complexes. In contrast, a significant level of HDAC3 was precipitated along with SMRT, which is in agreement with previous reports (Guenther et al. 2000; Li et al. 2000). Although SMRT also interacts with HDAC1 or HDAC2, these interactions are considerably weaker. Conversely, abundant HDAC1 and HDAC2 (but only minimal HDAC3) are present in the protein complexes associated with Atr2. Similarly, Atro, but not Atr1, also precipitates HDAC1/2 specifically, indicating that Atro-family (except Atr1) and SMRT-family proteins display distinct preferences for different HDACs.

Consistent with our HDAC assay results (Fig. 4B), removing the ELM2 domain or both the ELM2 and SANT domains from Atr2 $\triangle \mathrm{BAH}$ impairs or disrupts its ability to associate with $\mathrm{HDAC1} / 2$ (Fig. 4C, lanes 6,7). Given the fact that similar results were also obtained when we examined the distribution of endogenous HDAC1 in cells expressing different CFP-Atr2 variants (Supplementary Fig. 4), we therefore conclude that the ability of Atr2 to exert HDAC activity and to recruit HDAC1/2 depends on its ELM2 and SANT domains.

\section{Prospects and implications}

In many respects, the transcriptional properties that we discover here for Atro proteins parallel those found for SMRT, N-CoR, and SMRTER. As shown in Figure 5, (1) these two classes of corepressors share a SANT domain and RERE stretch; (2) they are conserved in vertebrates and in flies; (3) they bind NRs, albeit selectively; and (4) they associate with HDACs, also selectively. Additionally, Atr1, like SMRT and N-CoR, also interacts with ETO/MTG8 (Gelmetti et al. 1998; Wood et al. 2000), which is known to be a transcriptional repressor involved in acute myeloid leukemia. Considering that SMRT and N-CoR interact with a large number of NRs, with a wide variety of transcriptional factors, and also with type II HDACs, we predict that Atro proteins may

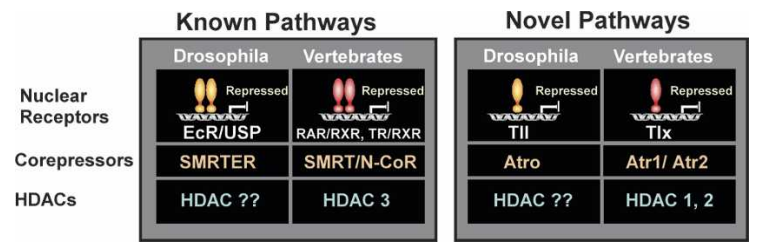

Figure 5. Comparing the properties of the SMRTER/SMRT/N-CoR class of corepressors and the Atro/Atr1/Atr2 class of corepressors. SMRT class corepressors preferentially bind NR1 proteins, such as $\mathrm{EcR}, \mathrm{TR}$, and RAR, and recruit HDAC3. Atro class corepressors selectively bind NR2 proteins, such as Tlx and Tll, and recruit HDAC1 and HDAC2. Note that COUP-TF and SVP1 can bind both classes of corepressors. have similar qualities as well. Therefore, more Atro-interacting factors still await discovery.

In the context of human diseases, it is known that polyglutamine expansion in human Atr1 causes DRPLA (Koide et al. 1994; Nagafuchi et al. 1994). We have shown that Atr1 lacks HDAC activity, yet it binds Atr2 through their RERE stretches (Yanagisawa et al. 2000), and it associates with both Tlx and COUP-TF, two known NRs with key roles in CNS development and functioning (Park et al. 2003; Roy et al. 2004; Shi et al. 2004). We therefore propose that mutant Atr1 may cause its pathological effects by interfering with the normal transcriptional properties of Atr2 and its associated nuclear receptors.

\section{Materials and methods}

\section{Antibodies}

Polyclonal Atro antibodies were developed in guinea pigs using KLHconjugated synthetic polypeptides, ADTPALRQLSEYARPHVA, which correspond to the 1815-1832 region of Atro. For all our experiments, we used affinity-purified Atro antibodies. The sources of other antibodies are anti-Tailless (gift of J. Reinitz, State University of New York-Stony Brook, New York), anti-Flag (Sigma F-7425; 1:400), anti-HDAC1 (Upstate 06-720; 1:500), anti-HDAC2 (Santa Cruz 7899; 1:400), anti-HDAC3 (Santa Cruz 11417; 1:150), anti-Sin3A (Santa Cruz 767; 1:300), Texas-Red conjugated anti-rabbit (Jackson ImmunoResearch 711-075-152; 1:400), and anti-Digoxigenin (Roche, 1333089; 1:1500). Immunofluorescence and microscopic analysis are described in Tsai et al. (2004).

Drosophila stocks and experiments

The $t 11^{1}, t 1 l^{e}$, and $h k b^{2}$ lines were obtained from the Bloomington Stock Center. The Atro $^{35}$ line was a gift of S. Kerridge (Centre CNRS-INSERMUniversité de la Méditerranée-AP de Marseille, France). Details for using the DFS-FLP method to generate Atro $^{35}$ germline clones are described in Erkner et al. (2002). The $t 11^{1}$, Atro ${ }^{35}$ double-mutant line was generated by chromosomal recombination. All genetic experiments were carried out at $23^{\circ} \mathrm{C}$. In situ hybridization experiments and the methods used to generate kni and ftz RNA probes are described in Tsai and Gergen (1994).

Cell culture, transfection, and coimmunoprecipitation experiments are described in Mizutani et al. (2005).

Additional materials and methods are included in the Supplemental Material.

\section{Acknowledgments}

We thank Ron Evans for nuclear receptor-expressing plasmids; S. Kerridge for Atro fly stocks; C. Ross and T. Waerner for Atr1 and Atr2 expressing plasmids; T. Xu, J. Reinitz, and L. Pick for reagents; the Bloomington Stock Center for Drosophila stocks; P. Gergen and X. Wang for advice on ChIP assays; and R. Head and J. Fondell for comments on this manuscript. This work is supported by the UMDNJ Foundation to C.-C.T.

\section{References}

Aasland, R., Stewart, A.F., and Gibson, T. 1996. The SANT domain: A putative DNA-binding domain in the SWI-SNF and ADA complexes, the transcriptional co-repressor N-CoR and TFIIIB. Trends Biochem. Sci. 21: $87-88$.

Boyer, L.A., Langer, M.R., Crowley, K.A., Tan, S., Denu, J.M., and Peterson, C.L. 2002. Essential role for the SANT domain in the functioning of multiple chromatin remodeling enzymes. Mol. Cell 10: 935 942.

Chen, J.D. and Evans, R.M. 1995. A transcriptional co-repressor that interacts with nuclear hormone receptors. Nature 377: 454-457.

Chou, T.B. and Perrimon, N. 1992. Use of a yeast site-specific recombinase to produce female germline chimeras in Drosophila. Genetics 131: 643-653.

Codina, A., Love, J.D., Li, Y., Lazar, M.A., Neuhaus, D., and Schwabe, 
J.W. 2005. Structural insights into the interaction and activation of histone deacetylase 3 by nuclear receptor corepressors. Proc. Nat1. Acad. Sci. 102: 6009-6014.

Erkner, A., Roure, A., Charroux, B., Delaage, M., Holway, N., Core, N. Vola, C., Angelats, C., Pages, F., Fasano, L., et al. 2002. Grunge, related to human Atrophin-like proteins, has multiple functions in Drosophila development. Development 129: 1119-1129.

Fanto, M., Clayton, L., Meredith, J., Hardiman, K., Charroux, B., Kerridge, S., and McNeill, H. 2003. The tumor-suppressor and cell adhesion molecule Fat controls planar polarity via physical interactions with Atrophin, a transcriptional co-repressor. Development 130: 763-774

Gelmetti, V., Zhang, J., Fanelli, M., Minucci, S., Pelicci, P.G., and Lazar, M.A. 1998. Aberrant recruitment of the nuclear receptor corepressorhistone deacetylase complex by the acute myeloid leukemia fusion partner ETO. Mol. Cell. Biol. 18: 7185-7191.

Guenther, M.G., Lane, W.S., Fischle, W., Verdin, E., Lazar, M.A., and Shiekhattar, R. 2000. A core SMRT corepressor complex containing HDAC3 and TBL1, a WD40-repeat protein linked to deafness. Genes \& Dev. 14: 1048-1057.

Guenther, M.G., Barak, O., and Lazar, M.A. 2001. The SMRT and N-CoR corepressors are activating cofactors for histone deacetylase 3. Mol. Cell. Biol. 21: 6091-6101.

Horlein, A.J., Naar, A.M., Heinzel, T., Torchia, J., Gloss, B., Kurokawa, R., Ryan, A., Kamei, Y., Soderstrom, M., Glass, C.K., et al. 1995. Ligand-independent repression by the thyroid hormone receptor mediated by a nuclear receptor co-repressor. Nature 377: 397-404.

Kankel, M.W., Duncan, D.M., and Duncan, I. 2004. A screen for genes that interact with the Drosophila pair-rule segmentation gene fushi tarazu. Genetics 168: 161-180.

King-Jones, K. and Thummel, C.S. 2005. Nuclear receptors-A perspective from Drosophila. Nat. Rev. Genet. 6: 311-323.

Koide, R., Ikeuchi, T., Onodera, O., Tanaka, H., Igarashi, S., Endo, K., Takahashi, H., Kondo, R., Ishikawa, A., Hayashi, T., et al. 1994. Unstable expansion of CAG repeat in hereditary dentatorubral-pallidoluysian atrophy (DRPLA). Nat. Genet. 6: 9-13.

Li, J., Wang, J., Nawaz, Z., Liu, J.M., Qin, J., and Wong, J. 2000. Both corepressor proteins SMRT and N-CoR exist in large protein complexes containing HDAC3. EMBO T. 19: 4342-4350.

Mahoney, P.A. and Lengyel, J.A. 1987. The zygotic segmentation mutant tailless alters the blastoderm fate map of the Drosophila embryo. Dev. Biol. 122: 464-470.

Mangelsdorf, D.J. and Evans, R.M. 1995. The RXR heterodimers and orphan receptors. Cell 83: 841-850.

Mizutani, A., Wang, L., Rajan, H., Vig, P.J., Alaynick, W.A., Thaler, J.P., and Tsai, C.C. 2005. Boat, an AXH domain protein, suppresses the cytotoxicity of mutant ataxin-1. EMBO J. 24: 3339-3351.

Nagafuchi, S., Yanagisawa, H., Ohsaki, E., Shirayama, T., Tadokoro, K., Inoue, T., and Yamada, M. 1994. Structure and expression of the gene responsible for the triplet repeat disorder, dentatorubral and pallidoluysian atrophy (DRPLA). Nat. Genet. 8: 177-182.

Pankratz, M.J., Hoch, M., Seifert, E., and Jackle, H. 1989. Kruppel requirement for knirps enhancement reflects overlapping gap gene activities in the Drosophila embryo. Nature 341: 337-340.

Pankratz, M.J., Busch, M., Hoch, M., Seifert, E., and Jackle, H. 1992. Spatial control of the gap gene knirps in the Drosophila embryo by posterior morphogen system. Science 255: 986-989.

Park, J.I., Tsai, S.Y., and Tsai, M.J. 2003. Molecular mechanism of chicken ovalbumin upstream promoter-transcription factor (COUPTF) actions. Keio J. Med. 52: 174-181.

Pignoni, F., Baldarelli, R.M., Steingrimsson, E., Diaz, R.J., Patapoutian, A., Merriam, J.R., and Lengyel, J.A. 1990. The Drosophila gene tailless is expressed at the embryonic termini and is a member of the steroid receptor superfamily. Cell 62: 151-163.

Privalsky, M.L. 2004. The role of corepressors in transcriptional regulation by nuclear hormone receptors. Annu. Rev. Physiol. 66: 315-360.

Roy, K., Kuznicki, K., Wu, Q., Sun, Z., Bock, D., Schutz, G., Vranich, N., and Monaghan, A.P. 2004. The Tlx gene regulates the timing of neurogenesis in the cortex. J. Neurosci. 24: 8333-8345.

Shi, Y., Chichung Lie, D., Taupin, P., Nakashima, K., Ray, J., Yu, R.T., Gage, F.H., and Evans, R.M. 2004. Expression and function of orphan nuclear receptor TLX in adult neural stem cells. Nature 427: 78-83.

Shi, Y.J., Matson, C., Lan, F., Iwase, S., Baba, T., and Shi, Y. 2005. Regu- lation of LSD1 histone demethylase activity by its associated factors. Mol. Cell 19: 857-864.

Strecker, T.R., Kongsuwan, K., Lengyel, J.A., and Merriam, J.R. 1986. The zygotic mutant tailless affects the anterior and posterior ectodermal regions of the Drosophila embryo. Dev. Biol. 113: 64-76.

Tsai, C.C. and Fondell, J.D. 2004. Nuclear receptor recruitment of histone-modifying enzymes to target gene promoters. Vitam. Horm. 68: $93-122$.

Tsai, C. and Gergen, J.P. 1994. Gap gene properties of the pair-rule gene runt during Drosophila segmentation. Development 120: 1671-1683.

Tsai, C.C., Kao, H.Y., Yao, T.P., McKeown, M., and Evans, R.M. 1999. SMRTER, a Drosophila nuclear receptor coregulator, reveals that EcR-mediated repression is critical for development. Mol. Cell 4: 175-186.

Tsai, C.C., Kao, H.Y., Mitzutani, A., Banayo, E., Rajan, H., McKeown, M., and Evans, R.M. 2004. Ataxin 1, a SCA1 neurodegenerative disorder protein, is functionally linked to the silencing mediator of retinoid and thyroid hormone receptors. Proc. Nat1. Acad. Sci. 101: 40474052.

Waerner, T., Gardellin, P., Pfizenmaier, K., Weith, A., and Kraut, N. 2001. Human RERE is localized to nuclear promyelocytic leukemia oncogenic domains and enhances apoptosis. Cell Growth Differ. 12: 201-210.

Wood, J.D., Nucifora Jr., F.C., Duan, K., Zhang, C., Wang, J., Kim, Y., Schilling, G., Sacchi, N., Liu, J.M., and Ross, C.A. 2000. Atrophin-1, the dentato-rubral and pallido-luysian atrophy gene product, interacts with ETO/MTG8 in the nuclear matrix and represses transcription. J. Cell Biol. 150: 939-948.

Yanagisawa, H., Bundo, M., Miyashita, T., Okamura-Oho, Y., Tadokoro, K., Tokunaga, K., and Yamada, M. 2000. Protein binding of a DRPLA family through arginine-glutamic acid dipeptide repeats is enhanced by extended polyglutamine. Hum. Mol. Genet. 9: 1433-1442.

Yu, R.T., McKeown, M., Evans, R.M., and Umesono, K. 1994. Relationship between Drosophila gap gene tailless and a vertebrate nuclear receptor Tlx. Nature 370: 375-379.

Yu, J., Li, Y., Ishizuka, T., Guenther, M.G., and Lazar, M.A. 2003. A SANT motif in the SMRT corepressor interprets the histone code and promotes histone deacetylation. EMBO J. 22: 3403-3410.

Zhang, S., Xu, L., Lee, J., and Xu, T. 2002. Drosophila atrophin homolog functions as a transcriptional corepressor in multiple developmental processes. Cell 108: 45-56.

Zoltewicz, J.S., Stewart, N.J., Leung, R., and Peterson, A.S. 2004. Atrophin 2 recruits histone deacetylase and is required for the function of multiple signaling centers during mouse embryogenesis. Development 131: 3-14 


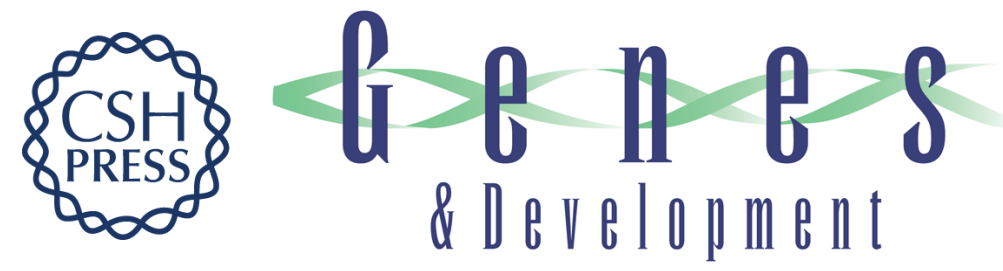

\section{Histone deacetylase-associating Atrophin proteins are nuclear receptor corepressors}

Lei Wang, Harini Rajan, Jeffrey L. Pitman, et al.

Genes Dev. 2006, 20:

Access the most recent version at doi:10.1101/gad.1393506

Supplemental http://genesdev.cshlp.org/content/suppl/2006/02/15/gad.1393506.DC1
Material

References This article cites 39 articles, 18 of which can be accessed free at: http://genesdev.cshlp.org/content/20/5/525.full.html\#ref-list-1

License

Email Alerting Receive free email alerts when new articles cite this article - sign up in the box at the top Service right corner of the article or click here.

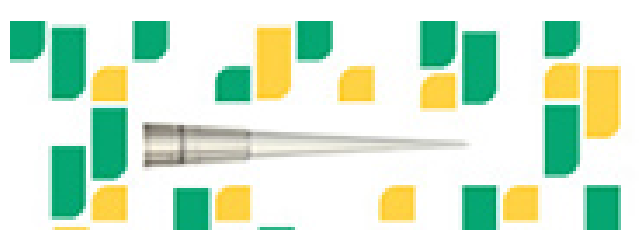

